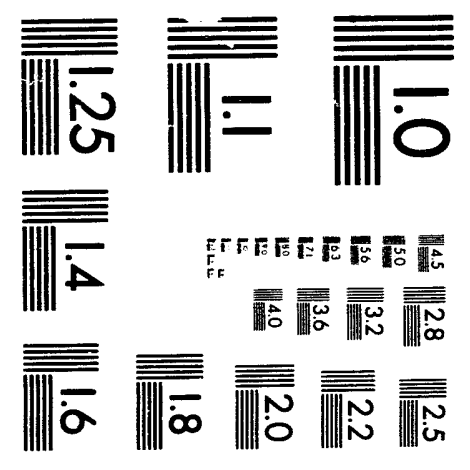



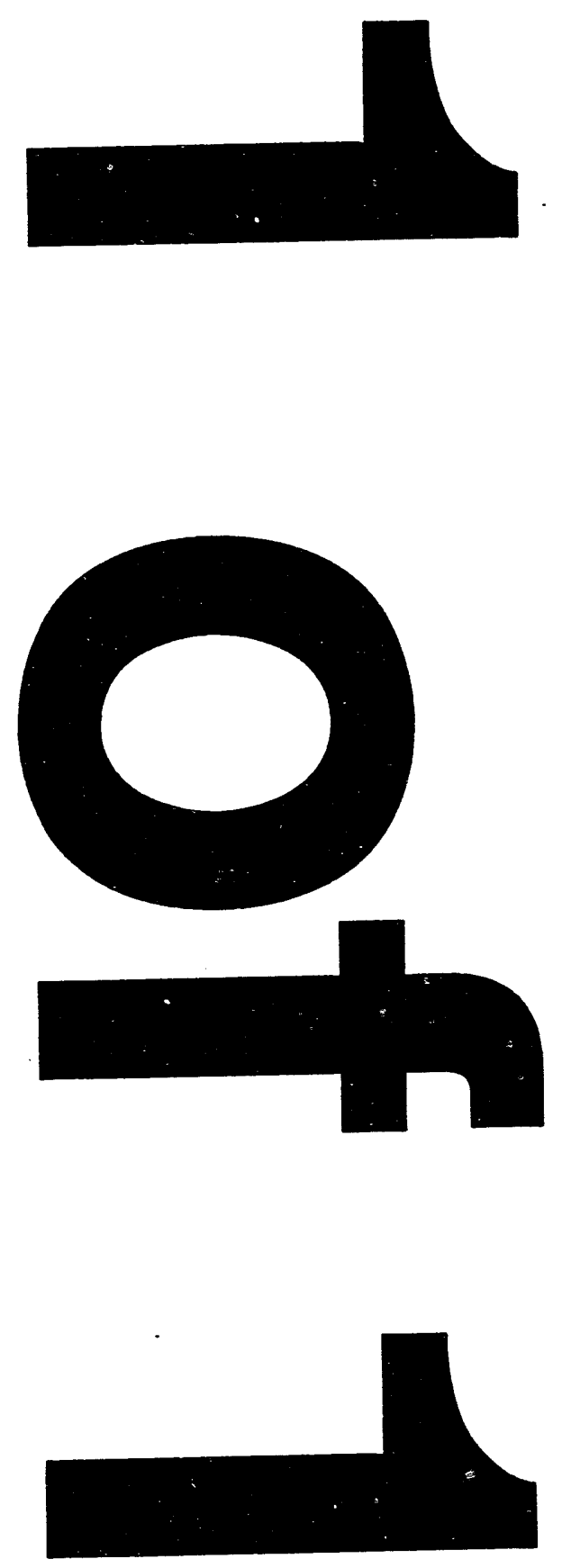
Title:

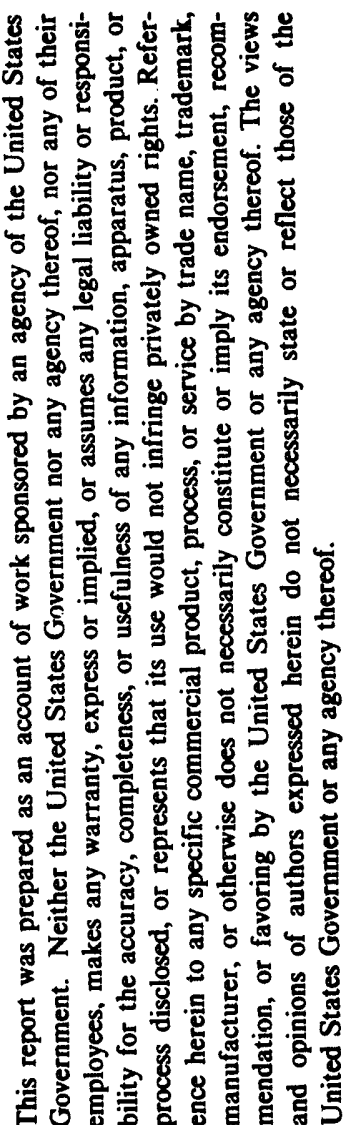

A 12-Channel VME-Based Pulse-Height Analysis Module

\section{DEC 131933}

Author(s): G. J. Arnone

OSTI
Submitted to:

IEEE 1993 Nuclear Science Symposium

October 31-November 4, 1993

San Francisco, California

\section{Los Alamos} NATIONAL LABOAATORY

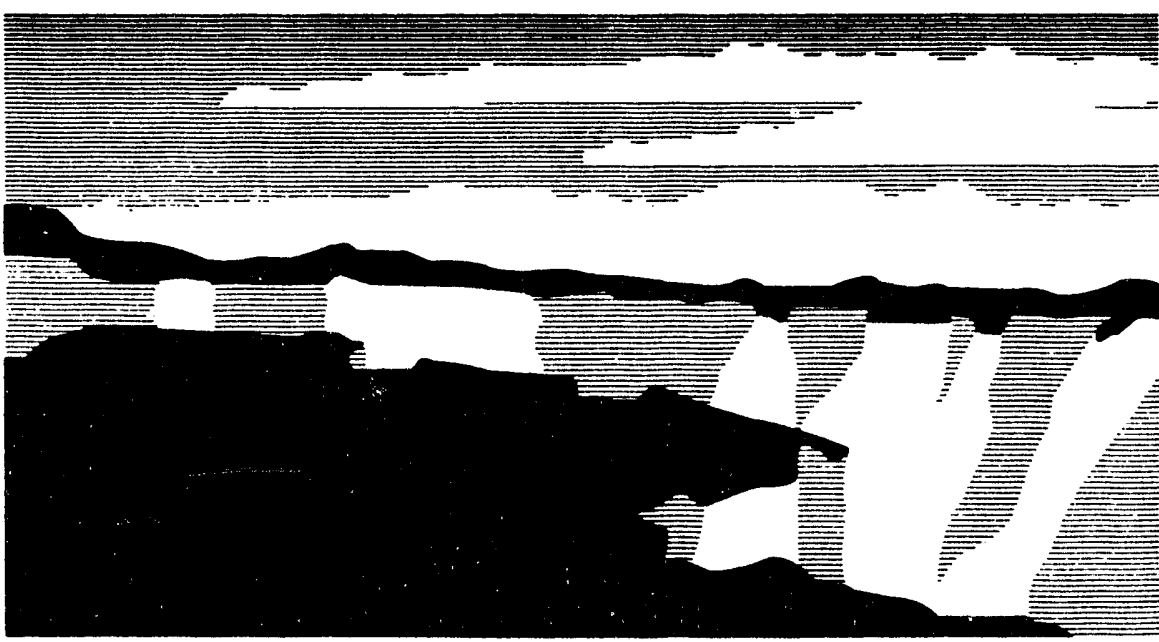

Los Alamos National Laboratory, an affirmative action/equal oppontunity emplofyer, is operated by the University of Calitornia for the U.S. Department of Energy under contract W.7405-ENG-36. By acceptance of this anticle, ithe publisher recognizes that the U.S. Government retains a nonoxctusive, royally-tres Hicense to publish or reproduce the publisthed form of this contribution, or to allow others to do so, for U.S. Government purposes. The Los Namos National Laboratory requests that the publisher identity this articte as work performed under the auspices of the U.S. Department of Enerpy. 


\author{
G. J. Arnone \\ Los Alamos National Laboratory, Los Alamos, New Mexico 87545
}

\begin{abstract}
We describe a 12-channel VMEbus-based pulse-height analysis board that was designed for use in a high-rate. multidetector. gamma-ray imaging system. This module was designed to minimize dead-time losses and to allow all key parameters to be software controlled. Gamma-ray detectors are connected directly to this module. eliminating the need for additional electronics.
\end{abstract}

\section{INTRODUCTION}

The need to instrument an array of 64 BGO detectors and provide very low dead-time and fast-processing capabilities led to the design of a VMEbus-based pulse-height analysis board, the VMEMCA. Several of these boards are used in the data acquisition system [1] developed for the nuclear resonance absorption project [2]. High single-channel instantaneous rates, up to $125-\mathrm{kHz}$, and low signal-to-noise ratios prompted us to design a module that could quickly process each overthreshold detector pulse. The charge-integration technique allows straightforward processing of each detector pulse without the need for charge preamps and shaping amplifiers. This technique also reduces the signal-processing time to the time it takes to integrate the charge from the photomultiplier tube (PMT). Another feature of the module is the dc-coupled analog signal path, which minimizes base-line shift.

Our BGO detectors, with an energy resolution of $\sim 15 \%$ for $137 \mathrm{Cs}$, enabled us to use 8-bit flash analog-to-digital converters (ADCs). This resulted in adequate en rgy resolution with fast processing times.

\section{VMEMCA MODULE OVERVIEW}

The VMEMCA board (Figs. 1 and 2) occupies 2 VMEbus slots and consists of 3 major sections: the VMEMCA motherboard, $12 \mathrm{MCA}$ plug-in boards, and a Mizar [3] MZ8135 CPU board. The VMEMCA motherboard is a $6 \mathrm{U}$ sized VME board that has the circuitry for interfacing the 12 MCA plug-in cards with the MZ8135 CPU board. The motherboard also contains

- the control digital-to-analog converters (DACs) that set the gain, the offset, the LLD, etc., of the MCA boards;

- the circuitry for generating over-threshold digital outputs; and

- the circuitry for driving activity indicators.

\footnotetext{
- This work was supported by the U.S. Federal Aviation Administration.
}

Each of the 12 MCA boards is a stand-alone. self-gated. pulse-height-analysis circuit that consists of the following:

- a programmable gain amplifier (PGA):

- a low-level discriminator (LLD):

- a charge integrator:

- an ADC: and

- a first-in-first-out (FIFO) memory.

The MZ8135 CPU board (a commercial. 3U-sized, 68030) CPU running at $40 \mathrm{MHz}$ with 1 megabyte of dual-ported memory) reads data from and controls the 12 MCA boards. Programs are down loaded into the CPU's memory, which reads and processes the data, making it available to processors on the VMEbus via its dual-ported memory.

The following relates briefly one channel's operation:

(1) When an input pulse from the detector goes over the LLD threshold, the charge integrator begins to integrate a delayed version of that pulse. (The integration time is software programmable from $100 \mathrm{~ns}$ to $3 \mu \mathrm{s}$.)

(2) Once the integration time has been completed, the 8-bit flash ADC digitizes the output level of the charge integrator and stores the data in the FIFO memory. The dead-time contribution, not including integration time, of the circuit is <100 ns, which is the sum of the digitization time and the integrator's reset time.

(3) Concurrent with step 2, the CPU section reads each channel's FIFO sequentially and processes data into histograms and regions of interest (ROIs). To icep up with the average input rate, the CPU must be able to process events at a rate of $300 \mathrm{kHz}$.

The parameters of the VMEMCA board can be changed under software control; they are the L.LD setpoint, the gain. the integration time, and the low- and high-level reference inputs of the ADC. The ability is pros the $A D C_{r}^{\prime \prime}$ reference inputs allows the operat... ir sctain cases. to increase the effective resolution of the ins coriversi-,

\section{SINGLE-ChANNEL MCA -AFC UTt G PERATION}

The single-channel MCA board isse (igs. and 4) uses all surface mount components pla 1 of is 4 - a $2 y, 7,4$ by $13-\mathrm{cm}$ printed circuit board. The followirs a dessixption of the MCA board's circuit operation.

Amplifiers UIA and UIB ampry the PMT signal AINPUT (the top trace of Fig. is a typical signal from $\mathrm{NaI}(\mathrm{Tl})$ detector after being amplitied by $\mathrm{U}: \mathrm{A}$ ]. The output of UIB is then fed to comparator U9A, which is used as the LLD and gate generator. The LLD, GAIN, INTIM, VREFL, and VREFH signals are obtained from DACs on the VMEMCA's motherboard. A pulse-processing cycle begins when an input signal goes over the threshold set by the LLD set point. U9B and U17 form a programmable-gate-width 


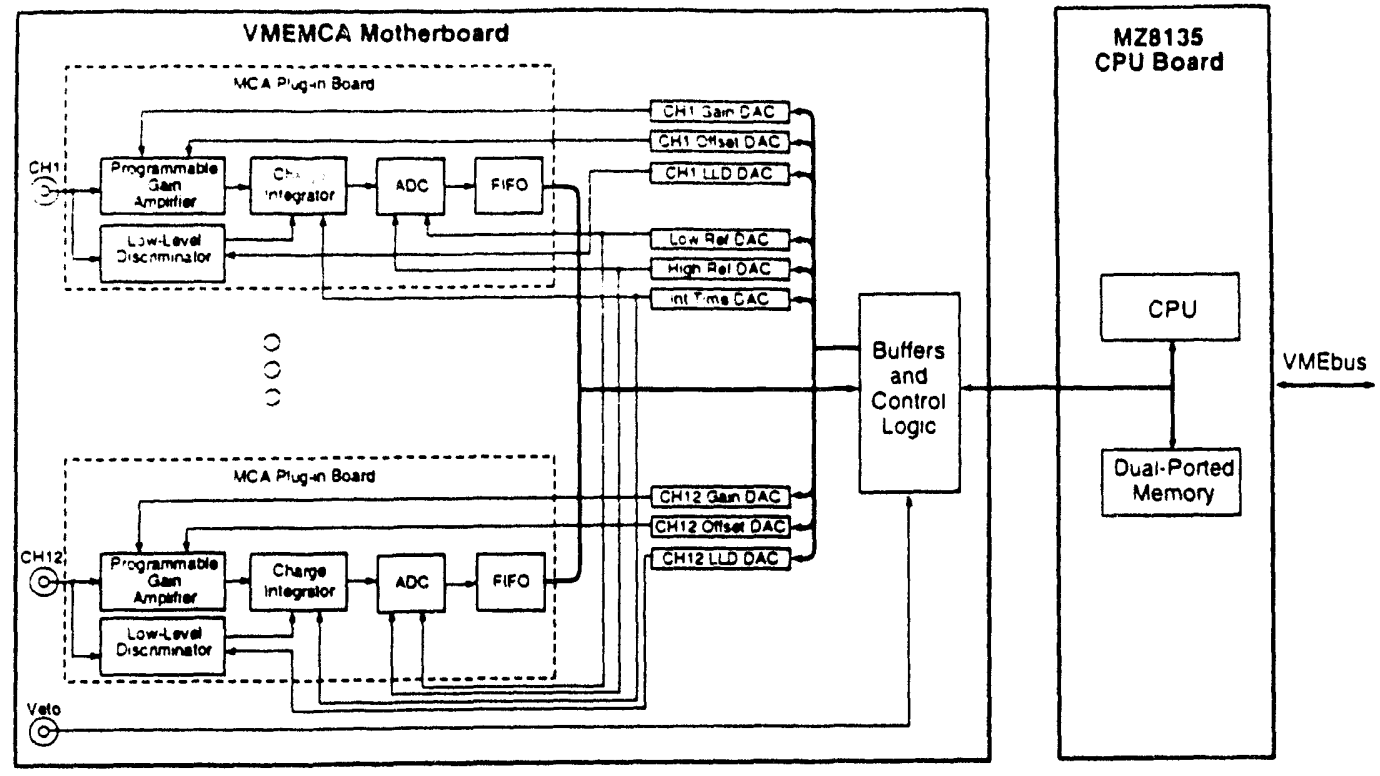

Fig. I. VMEMCA block diagram.

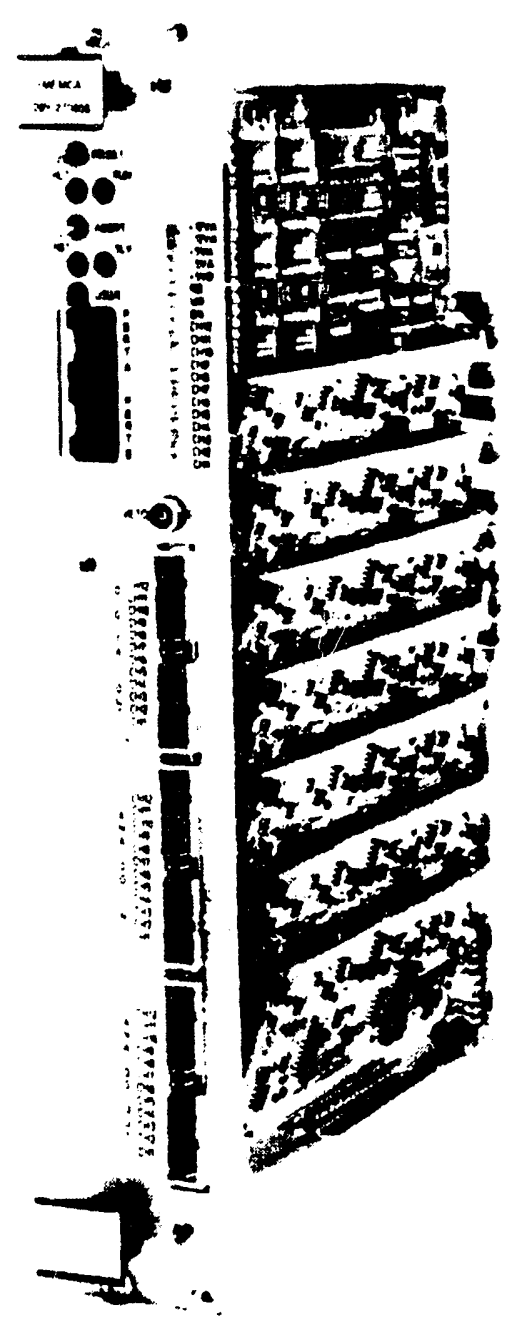

generator. When the input signal goes over threshold. Q5 is turned off, which allows C65 to start charging through constant current source U17. When the voltage at U9B pin 9 reaches the voltage set by INTIM, the pulse-processing cycle terminates. This action resets U9A via the latch input LE, thus enabling another cycle to begin when the next input signal goes over the threshold. During a processing cycle, the input pulse is amplified by UIA, then delayed by delay line U2, and fed to U3, which is a current mode multiplier that is used as the programmable-gain and offset stage. This device (U3) gives us a programmable signal gain range of 0.05 to 2.5. The output of $U 3$ then feeds through the analog switch U4A to U5, which is a FET input opamp used as the charge integrator. The output of the charge integrator is buffered by U16A; fed to the flash ADC (U6); then digitized at the end of the integration period. The ADC's data is loaded into FIFO U7. The ADC conversion is performed in $50 \mathrm{~ns}$, and the integrator settles in $\angle 50 \mathrm{~ns}$ after being reset. U16B provides a buffered linear output of the charge integrator for access on the front panel of the VMEMCA, (the bottom trace of Fig. 5 shows this output).

Fig. 2. VMEMCA photograph. 


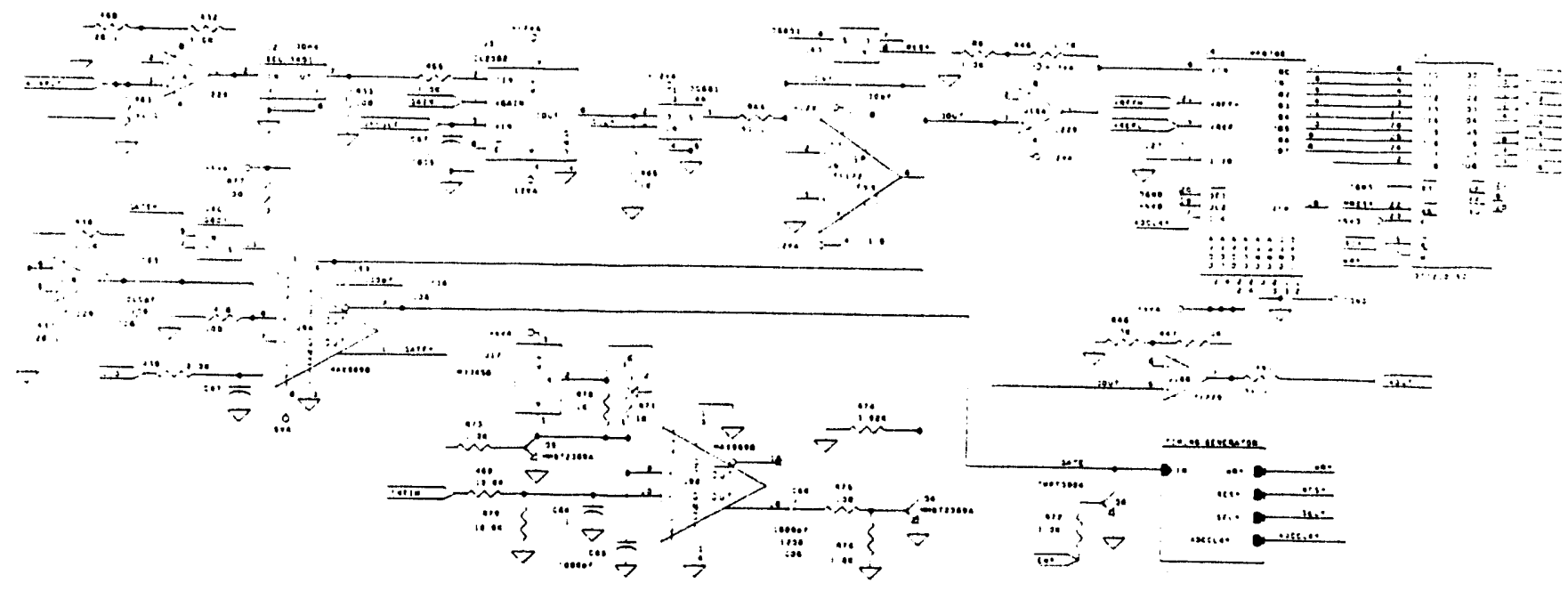

Fig. 3. Simplified schematic of MCA board.

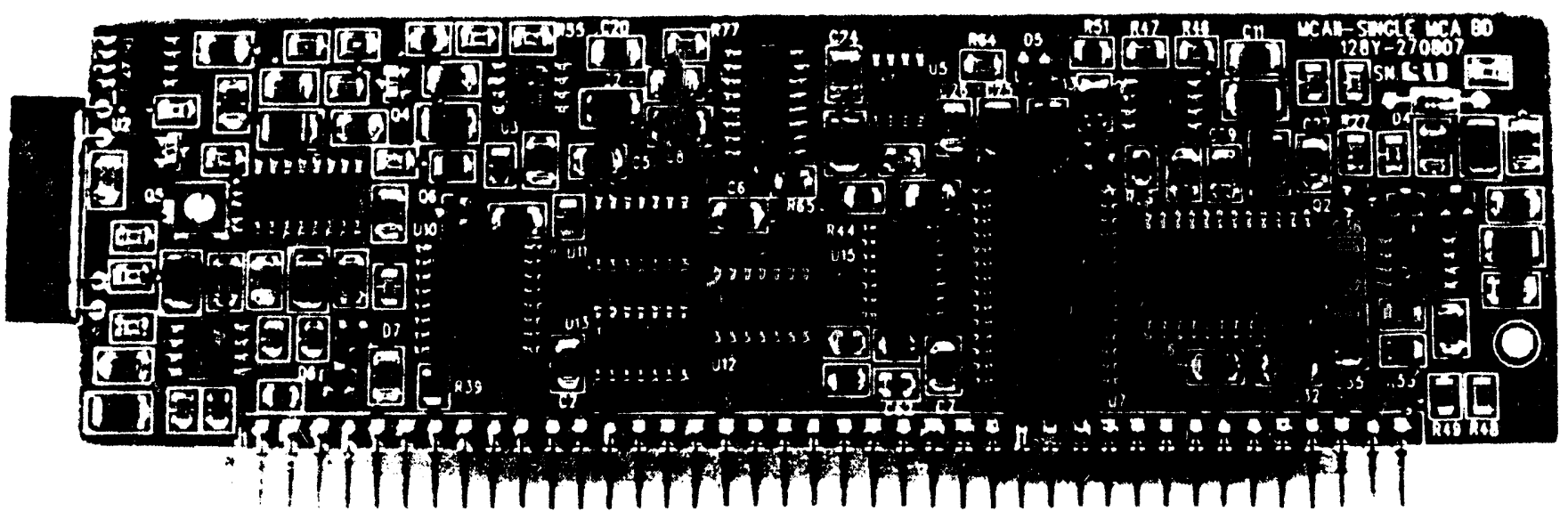

Fig. 4. MCA board photograph. 


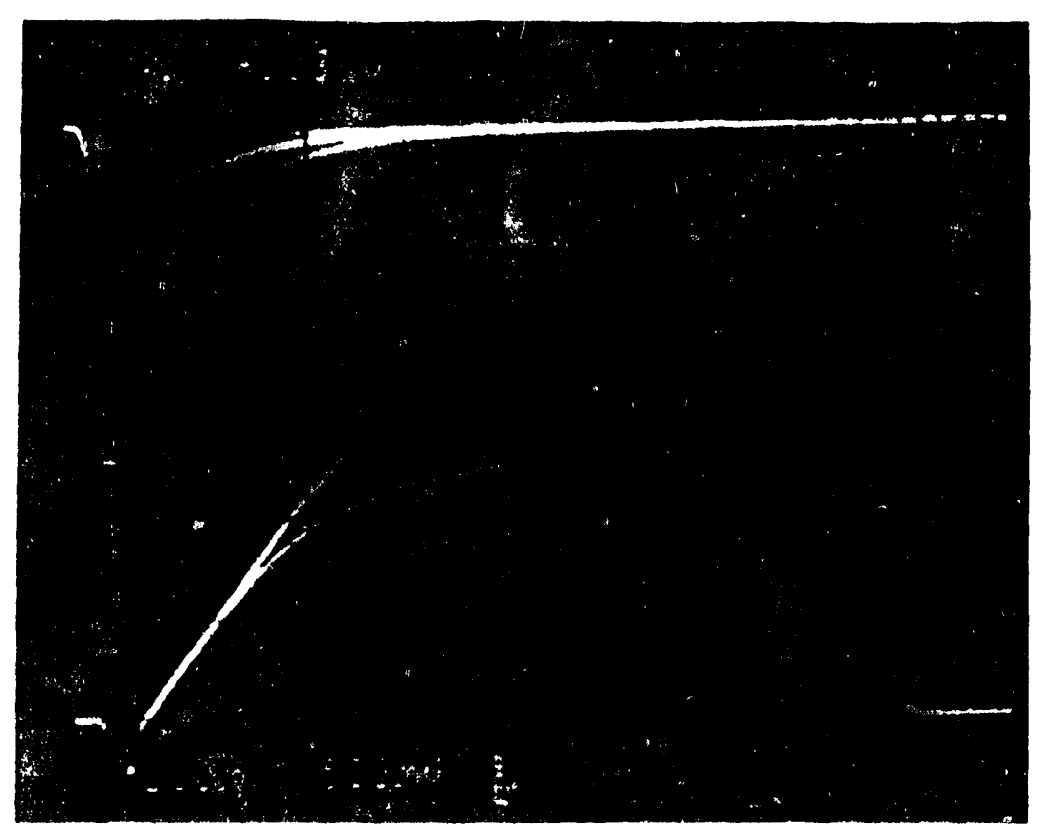

Fig. 5. Input signal (top trace) and integrator output signal (lower trace).

\section{VMEMCA PERFORMANCE}

We tested the module with isotopic sources such as $137 \mathrm{Cs}$ and ${ }^{60} \mathrm{Co}$ at average rates of $-50 \mathrm{kHz}$. Figure 6 shows a spectrum produced with these sources at that average rate. We obtained a $6.9^{\circ} \mathrm{C}$ resolution for ${ }^{137} \mathrm{Cs}$.

\section{CONCLUSION}

The VMEMCA module met all the design requirements for the project and is being used on another project using multiple $\mathrm{BaF}_{2}$ detectors. The modular design of the VMEMCA permits other functions, such as time-to-amplitude converters and higher resolution ADCs, to be implemented without redesigning the majority of the circuit. By using a commercial CPU and commercial real-time software, we delivered a working module in a short amount of time.

\section{ACKNOWLEDGMENTS}

I would like to thank Charles Hollas, LANL, for his invaluable input and assistance. I would also like to recognize Richard Morgado and Sam Gardner, LANL, for their efforts in pursuing this project.

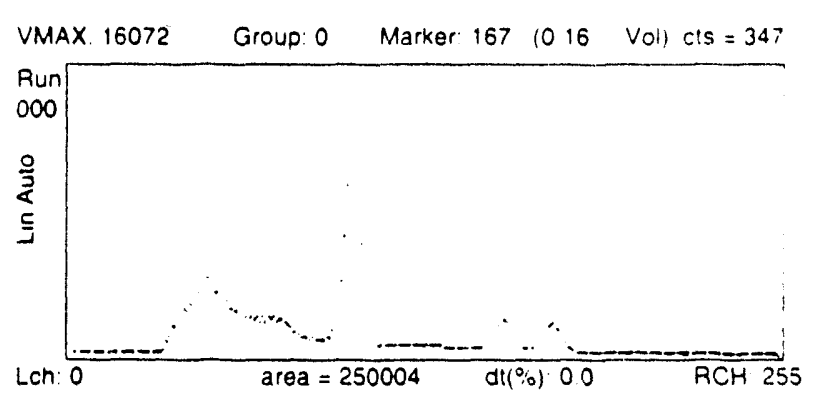

Fig. 6. Nal(Ti) detector spectrum.

\section{REFERENCES}

[1] G.J. Arnone and C.L Hollas. "Data Acquisition System Developed for the Resonance Absorption Project." to be presented at IEEE NSS. Nov. 1993.

[2] R.E. Morgado et. al., "Prototype Explosives Detection System Based on Nuclear Resonance Absorption In Nitrogen." (to be published).

[3] Mizar. 2410 Luna Road. Carrollton. Texas 75006 

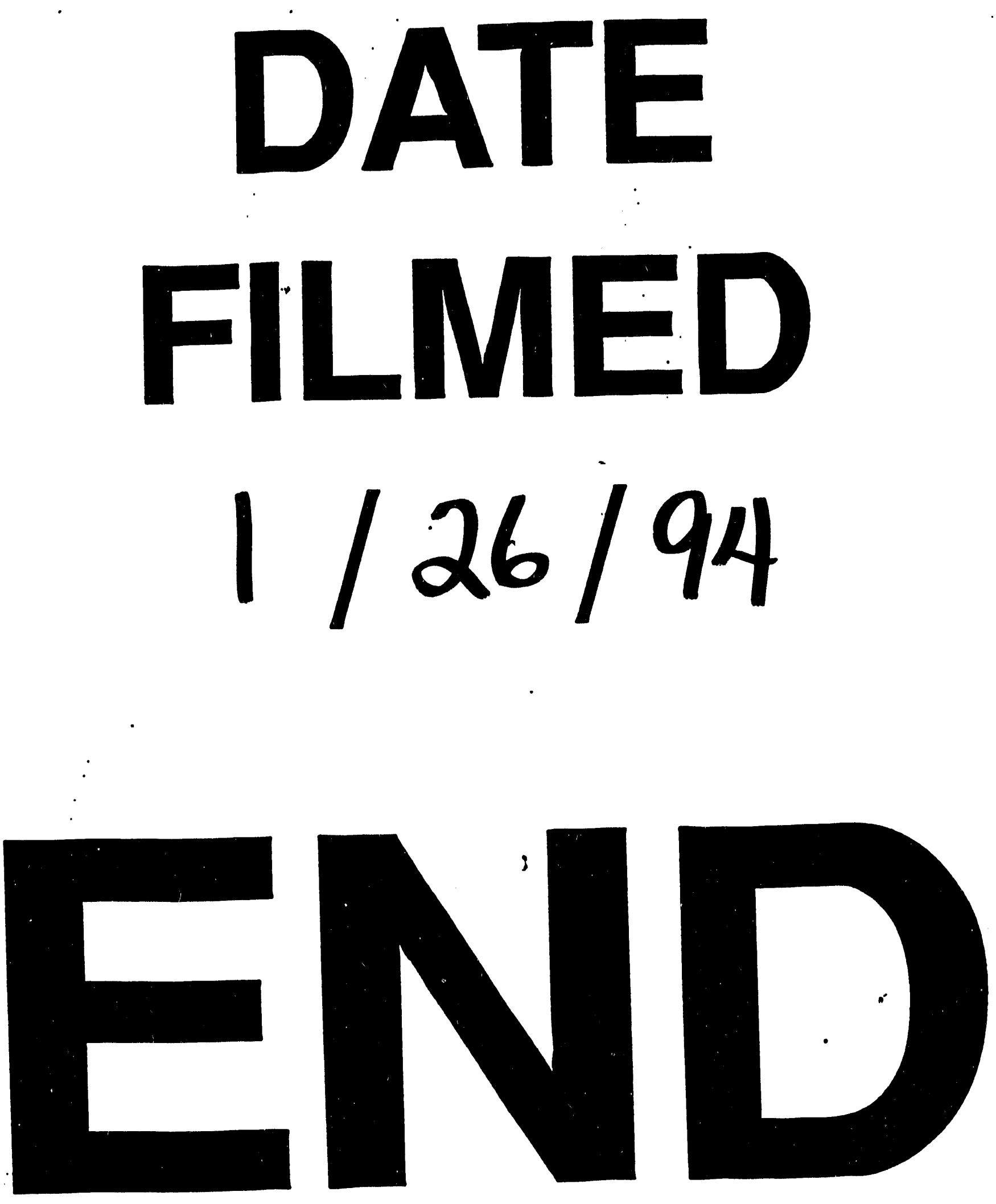
